\title{
Understanding Brain Mechanisms of Reactive Aggression
}

\author{
Katja Bertsch $^{1,2} \cdot$ Julian Florange $^{1} \cdot$ Sabine C. Herpertz ${ }^{1}$
}

Accepted: 26 October 2020 / Published online: 12 November 2020

(C) The Author(s) 2020

\begin{abstract}
Purpose of Review To review the current literature on biobehavioral mechanisms involved in reactive aggression in a transdiagnostic approach.

Recent Findings Aggressive reactions are closely related to activations in the brain's threat circuitry. They occur in response to social threat that is experienced as inescapable, which, in turn, facilitates angry approach rather than fearful avoidance. Provocation-induced aggression is strongly associated with anger and deficits in cognitive control including emotion regulation and inhibitory control. Furthermore, the brain's reward system plays a particular role in anger-related, tit-for-tat-like retaliatory aggression in response to frustration. More research is needed to further disentangle specific brain responses to social threat, provocation, and frustration.

Summary A better understanding of the psychological and neurobiological mechanisms involved in reactive aggression may pave the way for specific mechanism-based treatments, involving biological or psychotherapeutic approaches or a combination of the two.
\end{abstract}

Keywords Social threat · Provocation · Frustration · Threat sensitivity · Cognitive control · Emotion regulation · Inhibitory control · Frustrative non-reward

\section{Introduction}

Aggression is an evolutionarily highly conserved behavior directed toward another individual with the intent to cause harm [1]. Reactive aggression is commonly defined as a response to social threat, provocation, or frustration, and is strongly associated with anger $[2,3]$. Since increased reactive aggression is found in various mental disorders, it might be better regarded and explained as a transdiagnostic phenomenon. Reactive aggression is related to the following biobehavioral mechanisms: increased threat sensitivity [4] and frustrative non-reward $[5,6]$ as activating conditions as well as poor cognitive control as a regulatory condition $[4,7]$.

This article is part of the Topical collection on Personality Disorders

Katja Bertsch

Katja.bertsch@psy.lmu.de

1 Department of General Psychiatry, Center for Psychosocial Medicine, Medical Faculty, Heidelberg University, Heidelberg, Germany

2 Department of Psychology, Ludwig-Maximilians-University, Munich, Germany
Within the following three sections, we systematically summarize studies on reactive aggression from a transdiagnostic perspective by focusing on brain data that are associated with the three biobehavioral mechanisms of reactive aggression in healthy subjects and in clinical phenotypes associated with high reactive aggression. Since social threat, provocation, and frustration have been identified as the most important situational triggers for reactive aggression, we attempt to describe, and where possible disentangle, how the three biobehavioral mechanisms are related to these situations. However, it should be noted that a one by one assignment of the situational triggers and the biobehavioral mechanisms is neither intended nor possible. While explanations of the most important constructs and prevailing experimental paradigms that have been applied are included in the text, details about study designs, samples, sample sizes, and paradigms are provided in Table 1 (sorted by first author and year of publication). As can be seen in this table, we have selected studies published between 2013 and 2020, which included structural or functional magnetic resonance imaging (MRI), a measure for aggression, and a sample of ideally at least 30 participants (data that are based on smaller samples were only included if of particular relevance or if independent replication was available; for these 


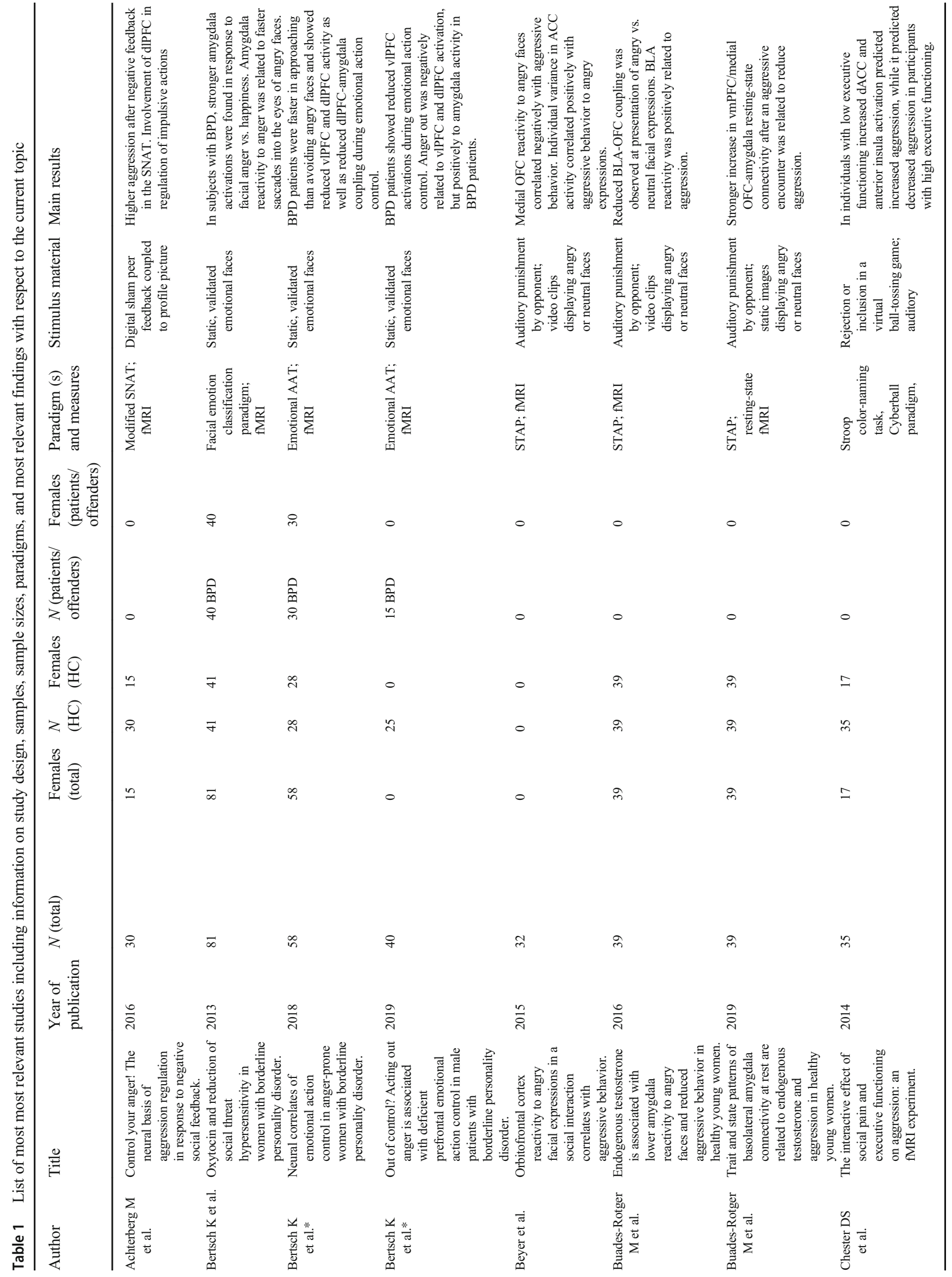




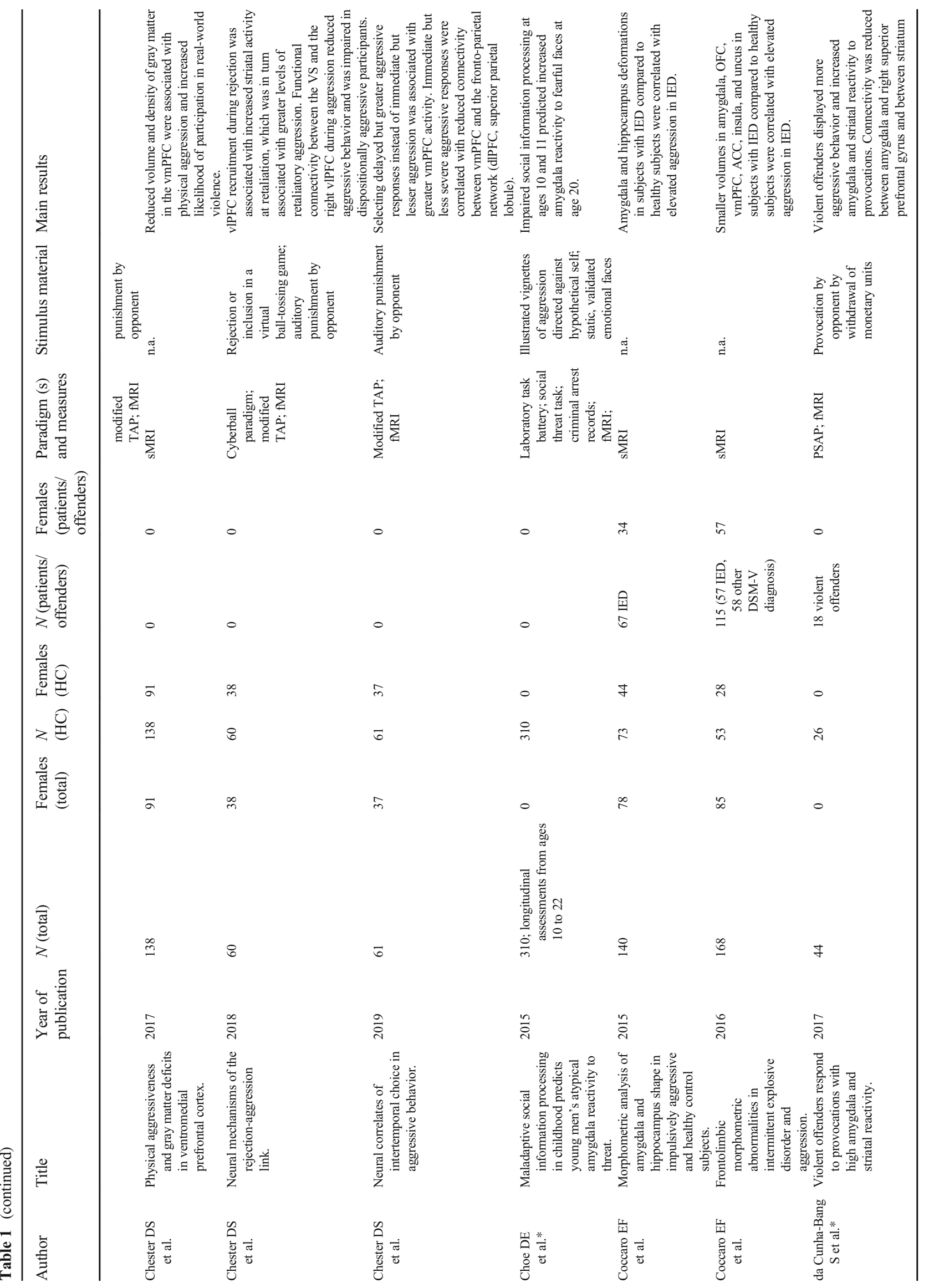




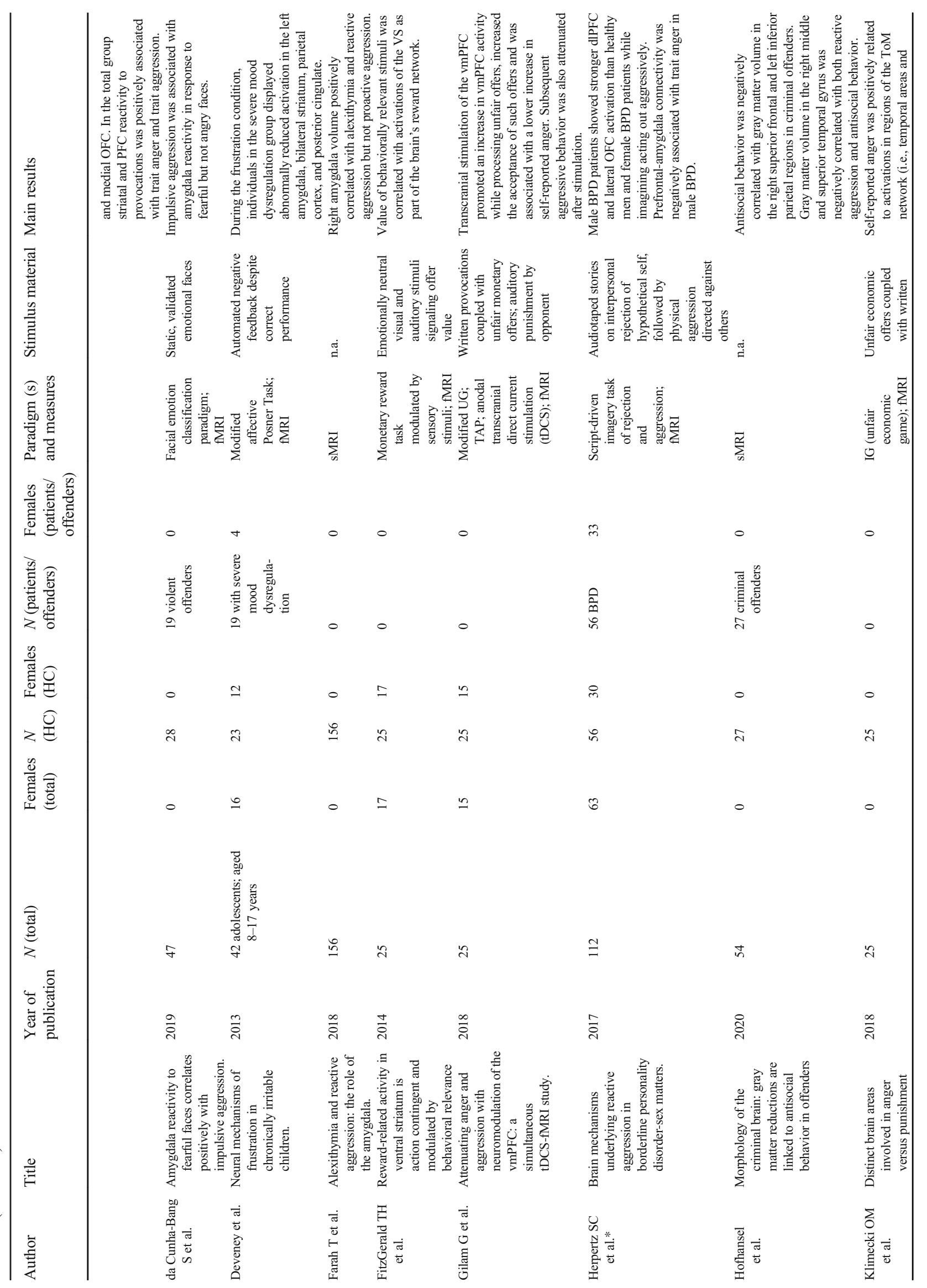




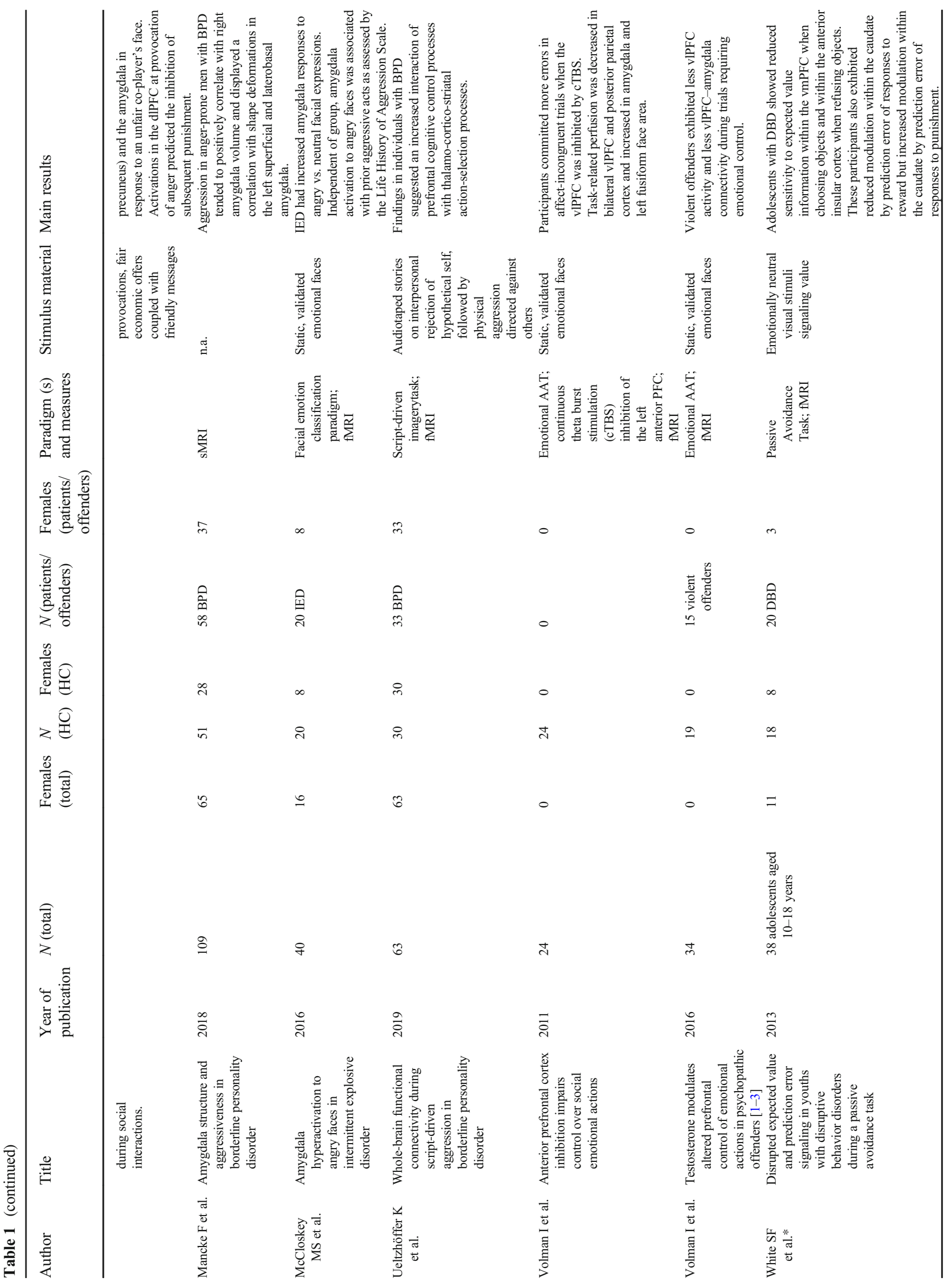




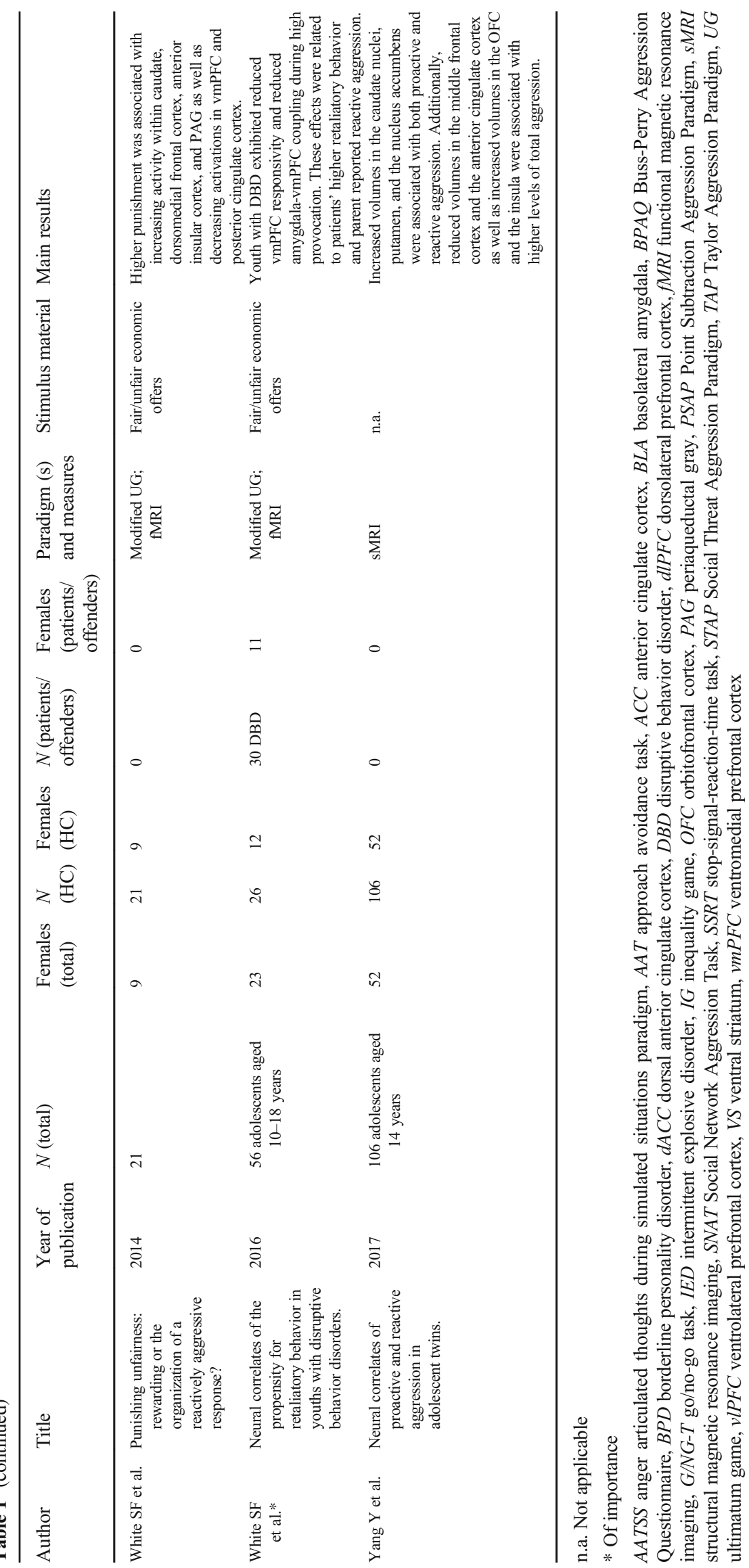


studies, $N$ is reported in the text). Detailed definitions of central constructs are provided in a supplementary glossary (see: https://doi.org/10.1007/s11920-020-01208-6). In the final section, we describe a working model and discuss limitations, open research questions, and clinical implications.

\section{Threat Sensitivity}

Threat elicits fear of harm followed by avoidance tendencies, but can also provoke reactive aggression [8-10]. In the animal model so-called defensive, fear-driven, hyperarousalassociated aggression has been extensively studied and occurs in situations challenging survival or defending limited supply in rivalry conditions [11] and is related to increased activity in the medial amygdala [12]. Since in rodents, this activity was positively related to aggression and associated automatic stress responses in a post-weaning social isolation model, this finding may point out a possible brain mechanism for human threat-related aggression in clinical groups with a history of childhood maltreatment [13].

In animals and humans, the likelihood of aggression in response to social threat depends on both situational and trait factors. Regarding situational factors, aggressive attack occurs in reaction to intense, unescapable social threats [10]. While Blanchard claims a distinct "fear of harm" phenotype of human aggression which is associated with the emotion of fear, others suggest that fearrelated escape behavior is likely to turn into angerrelated approach and, thus, aggressive behavior in the face of inescapable threat [8]. Whenever circumstances permit, individuals tend to calculate the value of response and gauge consequences of attack or escape in response to social threat with trait factors such as approach-avoidance tendencies moderating decision-making. While aggressive approach may be adaptive in the context of inescapable threat, individuals with high fear reactivity or strong avoidance tendencies are more likely to seek escape than to attack [14]. Particularly, clinical groups, most typically individuals with borderline personality disorder (BPD), tend to respond to even slight interpersonal challenge as though it was an inescapable threat [15], direct more attention to social threat cues [16], and show a hostile attribution bias [17], altogether increasing the likelihood for approach rather than avoidance behavior to potentially threatening cues. Importantly, in humans, situation and trait factors indissolubly interact: a threat cue may be perceived or interpreted as threatening by some but not by others. Particularly, clinical populations with a history of early maltreatment, as frequently found in BPD or antisocial personality disorder (ASPD), tend to be hypersensitive to social threat on the background of previous experiences of overwhelming threat that may trigger fight responses on the behavioral, neuronal, autonomic, and hormonal level [18]. Furthermore, fear and avoidance may turn into anger and approach behavior or individuals may rapidly oscillate between both emotions and action tendencies within situations they experience as threatening [8].

Core structures of the brain's threat circuitry subsume the amygdala, hypothalamus, and periaqueductal gray (PAG). The amygdala plays a key role in the processing of arousal and emotions including fear and anger. It facilitates behavioral, hormonal, and autonomic "survival" actions to imminent threat via the hypothalamus and PAG, while in the case of more distant or possibly escapable threat, it is likely to interact with prefrontal and striatal regions. In such situations, interactions of the amygdala with the ventromedial prefrontal cortex (vmPFC; involved in gathering action values and contingency information), the anterior insula (as part of the brain's salience network), and the anterior cingulate cortex (ACC; related to motor response selection), allow for a more thorough evaluation of the current threat situation and a cognitively controlled emotional and behavioral response [19]. Particularly, the vmPFC modulates the threat response, so that it is turned down when realizing that something - "at second sight"does not actually indicate danger, or is amplified in case of previously learnt contingencies are activated, as it happens in early stress related disorders [20]. Hence, childhood maltreatment, by affecting the development of the threat response system and its linkage with prefrontal areas, reprograms response to real or perceived threat cues in later life [20].

An association between reactive aggression and the amygdala is suggested by structural imaging data showing a positive correlation between reactive aggression and right amygdala volume in a large community sample [21]. Furthermore, smaller amygdala volume [22] and amygdala surface shape deformations [23] were related to elevated aggression in subjects with intermittent explosive disorder (IED), a disorder defined by reactive aggression. By contrast, larger volume and shape deformations of the amygdala correlated with aggression in anger-prone men with BPD [24].

In experimental studies, the threat circuitry is typically stimulated using pictures of fearful or angry faces. By communicating hostility, angry faces are used as a direct proxy for social threat [25], while fearful faces evoke attentional monitoring of a threatening environment and thus ambiguous threat [26]. In a large study in 10-12-year-old boys, the tendency to respond to ambiguous social scenes with hostility attribution and reactive aggression predicted participants' amygdala response to fearful faces in an emotional face matching task in young adulthood [27•]. This suggests an association between maladaptive social threat detection and aggressive responding [27-] and is consistent to a positive relationship between basolateral amygdala responses to angry faces and aggressive responses in healthy women undergoing the Social Threat 
Aggression Paradigm (STAP; [28]). In the STAP, a competitive reaction time task similar to the Taylor Aggression Paradigm (TAP), which however includes the presentation of brief video clips displaying an angry or neutral looking opponent just before the participant's punishment selection, amygdala activity was positively associated with aggression (i.e., the administered loudness of an aversive auditory punishment) in trials where the opponent showed angry but not neutral facial emotions [28]. Notably, in an inequality game (IG), self-reported anger was positively associated with amygdala activity when viewing the face of an unfair other who did not look angry but represented threat to the participant's selfworth by giving derogatory social feedback $(N=25$ healthy volunteers; [29]). In this competitive economic game, anger is induced by an unfair other player who gives derogatory feedback messages as opposed to a fair other player who behaves cooperatively.

Enhanced amygdala activity to angry faces and positive correlations between amygdala response and aggressive behavior in everyday life has been found in IED [30]. In BPD, amygdala reactivity was related to fast threat responses, i.e., faster saccades to the eyes of angry faces [16], which were in turn positively correlated with trait aggression [31]. A positive association was also found between trait aggression and amygdala reactivity to fearful faces in violent offenders [32].

Experimental provocation paradigms, such as the TAP, STAP, the Point Subtraction Aggression Paradigm (PSAP), or economic games (e.g., IG or ultimatum game (UG)), all comprise some forms of physical or monetary punishment or unfair treatment by another person within an interpersonal competition. Increment of activations found in the amygdala, hypothalamus, and PAG in a number of studies using these game paradigms might suggest that the brain's threat response system is involved in provocation [19]. In line with this, in healthy individuals, PAG activation increased with higher levels of punishment in an UG, which creates provocation in form of unfair offers ( $N=21$ healthy volunteers; [33]). Enhanced amygdala activity to provocation has also been reported in highly aggressive groups. For instance, in adolescents with disruptive behavior disorders (DBD), amygdala and PAG responses to provocation correlated positively with retaliatory behavior in the UG [34•]. Furthermore, violent offenders showed increased amygdala response to provocation in the PSAP, where aggression is provoked by a fictitious opponent stealing points from the participant's account. Amygdala activity correlated positively with task-induced aggressive behavior that is stealing points without gaining anything for oneself [35]. Notably, not all studies have found amygdala activations in response to provocation [36]. Discrepant findings may simply represent type II error or be attributable to task-related differences in amygdala habituation or cognitive demands.

\section{Frustrative Non-reward}

Frustrative non-reward is a further trigger for anger and reactive aggression, subsuming reactions elicited by prevented or withdrawn rewards. As such, frustrative non-reward can be understood as an expectancy violation that is the outcome of a negative prediction error [37]. The core region mediating frustrative non-reward is the ventral striatum (VS), which acts in conjunction with the vmPFC, insula, dorsal ACC, and inferior frontal gyrus (IFG) [38]. The VS, as part of the brain's reward network, is involved in learning about the predictive reward and reinforcement contingencies of (social) signals ( $N=25$ healthy volunteers; [39]).

Aggression in response to frustration typically occurs when one's desired goal is blocked [40]. It has been suggested that retaliatory behavior, accompanied by feelings of revenge, may compensate for frustrative non-reward by enhancing VS activity ("sweetness of revenge"). For instance, violent offenders' enhanced striatal activity was found to positively correlate with trait aggression and anger in the PSAP [35]. Furthermore, higher VS activity was reported in the decision phase of a competitive task similar to the TAP in which the loser was punished by an aversive noise and the intensity of the blast was determined by the opponent [41]. VS activity predicted the degree of retaliatory behavior in the game and was associated with the participant's life history of violent behavior [41]. Activations in competitive tasks often involve not only the ventral but also the dorsal striatum (caudate nucleus), which mediates the selection and preparation of motor responses (e.g., retaliatory punishment). In an UG, increasing activity in the dorsal striatum was related to higher punishments of frustrative offers in healthy volunteers $(N=21$ healty volunteers; [33]).

Furthermore, brain imaging data suggests that reactive aggression resulting from frustration and provocation is modulated by control areas of the vmPFC and lateral prefrontal cortex (PFC). The vmPFC mediates processing reward vs. punishment for regulatory purposes in conflicting decisionmaking, with an impact on automatic and instrumental action tendencies [19]. Correspondingly, enhanced acceptance of unfair offers and dampened anger response in the UG were found after transcranial stimulation of the vmPFC $(N=25$ healthy volunteers; [42]), and physical aggression correlated with smaller vmPFC [43] but larger striatal [44] volume in healthy participants. Additionally, retaliatory aggression in response to social exclusion was associated with VS activity and connectivity to the ventrolateral prefrontal cortex (vlPFC) [45]. Connectivity was reduced in aggressive participants, consistent with the vIPFC's role in emotional action regulation [45].

An involvement of a dysregulated threat network along with abnormal activities in reward structures in response to frustration is suggested by some clinical studies. In youth with 
$\mathrm{DBD}$, frustrative non-reward induced by highly unfair offers in an UG was associated with enhanced amygdala and PAG activity and, thus, a hyperactive threat circuit, as well as reduced vmPFC-amygdala connectivity. Interestingly, this neuronal pattern was correlated with task-induced retaliatory behavior and parent-reported reactive aggression [34•]. Youths with DBD also showed reduced use of expected value information within the vmPFC in a decision-making task where reinforcement was probabilistic that is over the course of the experiment, two cues were followed by feedback of gain and two other cues by feedback of loss [46•]. In this study, youths with DBD also showed enhanced responsiveness to frustration, namely, negative prediction errors (punishment was greater than expected) during feedback which was processed in the caudate [46•]. However, in a study of youth with disruptive mood dysregulation disorder (DMDD), frustrative non-reward was not associated with increased amygdala activity but, on the contrary, with decreased amygdala activity in response to negative social feedback in a cued-attention task performed under frustrating and non-frustrating conditions [47].

\section{Cognitive Control}

Social threat, provocation, and frustration trigger negative feelings, such as anger and a behavioral impulse to an aggressive approach. However, oftentimes, individuals do not act out aggressively in such situations. Here, cognitive control, a set of higher-order regulatory functions primarily mediated by the prefrontal cortex [48] that support goal-directed behavior by modulating other cognitive and emotional processes, comes into play [49]. So far, empirical evidence from both neuroscience and clinical studies particularly underlines deficits in two cognitive control functions, namely, inhibitory control and emotion regulation $[50,51]$. While inhibitory control or behavior control implies the inhibition of fast, premature, uncontrolled behavioral responses to pursue goaldirected behavior [48], emotion regulation or emotion control is defined as the ability to downregulate negative emotions [52]. In aggressive situations, dysregulated emotions aggravate poor behavior control. Thus, both, behavior and emotion control, are mediated by a broad fronto-parieto-insular network of brain regions implicated in monitoring, selecting, modulating, and evaluating behavioral/emotional responses, namely the dorsomedial prefrontal cortex (dmPFC)/dorsal anterior cingulate cortex (dACC)/pre-supplementary motor area (preSMA), bilateral anterior insula reaching into the IFG/ vlPFC, and inferior parietal lobules (IPL).

Very recently, negative associations between gray matter volume in regions belonging to this cognitive control network (i.e., right super frontal gyrus, right middle and superior temporal regions, and left inferior parietal lobe) and antisocial behavior - and for the right temporal lobe - reactive aggression have been reported in male criminal offenders [53]. Furthermore, stronger activations in parts of this system have been found in response to high vs. low levels of provocation or videos showing an angry vs. neutral looking opponent with most consistent activations in the dmPFC and dACC across functional neuroimaging studies [36]. In two studies, healthy participants showed elevated activations in $\mathrm{dmPFC} / \mathrm{dACC}$, IFG, and superior temporal gyrus in response to angry vs. neutral looking opponents in the STAP [28, 54]. These regions have been implicated in monitoring conflicts between desired and actual actions (dmPFC), response monitoring and error processing (dACC together with anterior insula), and the selection of context-appropriate and inhibition of contextinappropriate response (IFG/vIPFC together with inferior parietal lobules [55]). In close interaction with the inferior parietal lobules (IPL), the IFG is involved in modulating excitatory circuits of preSMA and subthalamic nucleus, thus leading to enhanced inhibition from the subthalamic nucleus to the motor cortex [56] and to subcortical regions implicated in automatic, stereotyped emotional action impulses, i.e., the amygdala, VS, hypothalamus, and PAG. Correspondingly, choosing an aggressive response for an opponent in the STAP involves a set of cognitive control processes and response selection. High levels of social threat raised by the opponent's angry expression and high provocation may intertwine in increasing the demand of inhibiting the urge to act out impulsively during the STAP in normal volunteers [28].

When having to inhibit fast amygdala-driven emotional action tendencies in approach-avoidance tasks, aggressionprone patients with BPD [57•, 58•] and violent offenders [59] were found to show lower recruitment of the vIPFC and dlPFC, which has been related to the tendency to act out anger [58•]. In these tasks, participants are instructed to either approach or avoid an angry or happy facial expression by pulling or pushing a joystick. Inhibiting fast tendencies to approach happy and avoid angry faces in order to perform the opposite behavior has been consistently related to an increase in lateral PFC activations in healthy volunteers [60]. The inhibition of vlPFC activity with transcranial magnetic stimulation resulted in worse performance and increased amygdala activity in healthy male participants. This supports the vlPFC's direct involvement in emotional action control and response selection by integrating and coordinating different cognitive processes $(N=24$ healthy volunteers; [61]), while the dlPFC has been associated with keeping control strategies and goals in mind and directing attention to relevant perceptual inputs, a continuous updating and manipulation of stimuli in working memory, and emotion regulation $[62,63]$. In another study, in which healthy participants adjusted the intensity of an aversive noise blast after receiving negative, positive, or neutral feedback from an alleged co-player, elevated right dlPFC activation to negative feedback was related to lower levels of 
retaliation [64]. Furthermore, male patients with BPD showed stronger dIPFC and lateral orbitofrontal cortex (OFC) activations compared to controls while imagining acting out aggressively [65•]. Since the prefrontal-amygdala connectivity of men with BPD was negatively associated with trait anger [65•], this suggests poor top-down adjustment despite efforts of control. Here, participants were instructed to vividly image brief stories describing neutral situations, anger-inducing interpersonal rejections, and subsequent acts of physical aggression against the provocateur. In the same study, female patients with BPD compared to healthy controls showed stronger increase in connectivity within a large brain network that suggested increased interaction of prefrontal cognitive control processes with thalamo-cortico-striatal action-selection processes while processing aggressive actions [66]. A stronger increase in connectivity between regions of the cognitive control network and regions of the motor system might be interpreted as the patients' attempts to control aggressive action impulses. These results may thus reflect a tendency to try and compensate ineffective emotion regulation strategies by directly suppressing aggressive action impulses [66].

There are also reports of reduced functional coupling between the amygdalae and the vmPFC/medial OFC (mOFC) in response to angry faces in healthy individuals [28], suggesting differences in the coupling between threat system (amygdalae) and contingency processing ( $\mathrm{vmPFC} / \mathrm{mOFC})$. As mentioned above, individuals are required to choose a more or less aggressive response for their opponent while facing this person's threatening expression. In healthy individuals, a stronger increase in $\mathrm{vmPFC} / \mathrm{mOFC}$-amygdala resting-state connectivity after the STAP was related to reduced aggression [67]. Furthermore, selecting delayed but greater aggressive responses (i.e., louder noise blast) instead of immediate but lesser aggression (i.e., softer noise blast) in a modified TAP was associated with greater vmPFC activity and an elevated connectivity with the fronto-parietal network in healthy subjects [68], probably reflecting higher cognitive load when thinking about contingencies [19]. These reports are accompanied by reports of reduced gray matter volumes in the vmPFC/OFC, ACC, amygdala, insula, and uncus in individuals with IED which were correlated with measures of aggressive behavior [22] and reports of severe deficits in behavior and emotion control in individuals with prefrontal lesions $[69,70]$.

As noted above, healthy participants showed stronger dACC activations in trials with high vs. low levels of provocation across studies [36] in line with this region's involvement in emotion regulation, response monitoring, and error processing. Classically, the so-called Go/No-Go tasks, which require a response to certain frequency and a response inhibition to infrequent stimuli, have been used to investigate behavior control. During such a task, deficient error processing, in terms of smaller event-related EEG potentials generated in the $\mathrm{dACC}$, has been reported in highly aggressive individuals
[71], and strong associations between trait anger and deficits in inhibitory control and automatic error processing were found in forensic patients during hostile conditions of an affective Go/No-Go task [72]. Furthermore, the interaction of behavior control assessed in a color-naming Stroop task and $\mathrm{dACC} /$ anterior insula response to social rejection in a Cyberball game predicted reactive aggression in healthy participants [73]: While individuals with low behavior control showed a positive association between insula/dACC activations and aggression, this association was negative in individuals with high behavior control. The results hence indicate an interference of negative emotional states triggered by provocation, rejection, and other negative interpersonal experiences with cognitive functions in individuals with low inhibitory control, which may lead to impulsive aggressive behavior. This is in line with evidence from the IMAGEN consortium on neural correlates of behavioral inhibition in 1709 participants according to which the lateral OFC is particularly involved in the processing of stop signals that inhibit actions, the dIPFC in attentional processes influencing task performance, and the anterior insula and ACC in emotional processes related to failure [74].

Thus, reactive aggression is likely to occur in individuals in whom poor inhibitory control co-occurs with deficient emotion regulation. Refraining from aggressive actions when socially threatened, provoked, or frustrated requires the capacity to downregulate negative emotions and inhibit the urge to act outs aggressively. The stronger the elicited emotions, the higher the need for cognitive control. Notably, cognitive control is not always reliable and/or tends to become fatigued with frequent use, rendering some individuals prone to aggressive acts. Selfreport data confirm deficits in inhibitory control [75-77] and in emotion regulation [78-80] in highly aggressive individuals who also seem to benefit less from explicit instructions to down-regulate negative emotions with the so-called adaptive strategies, such as reappraisal or distancing [81]. While for patients with BPD, in whom aggression has been shown to only occur in highly emotional contexts, emotion dysregulation is of particular importance for reactive aggression, deficient behavior, and emotion control might be equally relevant in ASPD and IED [51]. On a neural level, social threat, provocation, or frustration may challenge the core fronto-parieto-insular network implicated in behavior and emotion control. In these situations, fast action tendencies of approach or avoidance driven by subcortical circuits dominate rendering vulnerable subjects prone to act out on the urge to aggress. Results from functional and structural and lesion studies suggest a particular relevance of the PFC in pursuing goal-driven behavior maybe by sending bias signals to other brain areas that favor relevant sensory input, memories, and motor output [48]. 


\section{Working Model and Open Research Questions}

We systematically summarized and structured the recent literature in three sections on threat sensitivity and frustrative nonreward as activating conditions and cognitive control subsuming regulatory mechanisms to search for transdiagnostic biobehavioral mechanisms. Based on these sections, we will formulate a working model for future research designs trying to capture the multidimensional nature of reactive aggression and hypothesizing about pathways from the typical situational triggers social threat, provocation, and frustration, via the three brain mechanisms, to behavior (Fig. 1). First, though, we would like to acknowledge some important limitations: (i) data on neural correlates of induced reactive aggression, particularly in highly aggressive or clinical groups, are still limited, which may be strongly related to (ii) difficulties in reliably inducing reactive aggression in the MRI environment. (iii) Existing studies revealed an array of activated brain regions which have been interpreted in various ways based on a priori hypotheses, the particular paradigm used, or the specific functional deficits of the included clinical group. Different task demands which may also explain various activation patterns have not fully been addressed in the current review. (iv) Although we focused on studies in which aggression is explicitly measured, the heterogeneity and variation in the symptomatology of clinical phenotypes need to be taken into account, in addition to the correlational nature of the presented brain imaging results. Together with well-known limitations of MRI in terms of temporal and spatial resolution, small samples of patients with heterogenous clinical disorders may tremendously limit the interpretability of available data. (v) Social threat, provocation, and frustration share important aspects, since all three elicit negative emotions and they can interact with one another. (vi) The selected conditions are not exclusive and many more processes may be involved in the activation and regulation of aggressive reactions, such as decision-making, theory-of-mind, or empathy. (vii) The current review does not address developmental aspects, or genetic and epigenetic mechanisms of reactive aggression [82-84]. It neither considers effects of sex hormones nor of vasopressin and oxytocin, although first promising effects on social threat, provocation, and frustration processing have been reported $[16,85-88]$. For these reasons, the following working model should be regarded as a hypothesis to stimulate further research.

The brain's threat network comprises the amygdala, PAG, and hypothalamus. An individual's threshold for detecting social threats may be lowered by attentional threat biases or hostile attributions associated with faster, elevated, and/or prolonged responses of the threat circuity. Depending on individual differences and the proximity of the threat, a fronto-parieto-insular cognitive control network, in particular brain regions supporting emotion regulation and inhibitory control, may exert modulatory effects. Since economic game tasks that have been designed to
Fig. 1 Working model of reactive aggression representing major brain regions underlying threat sensitivity and frustrative nonreward as activating conditions and cognitive control as regulating condition of reactive aggression. AMY, amygdala; $\mathrm{CN}$, caudate nucleus; HYP, hypothalamus; dACC, dorsal anterior cingulate cortex; dIPFC, dorsolateral prefrontal cortex; dmPFC, dorsomedial prefrontal cortex; IFG, inferior frontal gyrus; INS, insula; IPL, inferior parietal lobules; $\mathrm{mOFC}$, medial orbitofrontal cortex; PAG, periaqueductal gray; vlPFC, ventrolateral prefrontal cortex; vmPFC, ventromedial prefrontal cortex; VS, ventral striatum

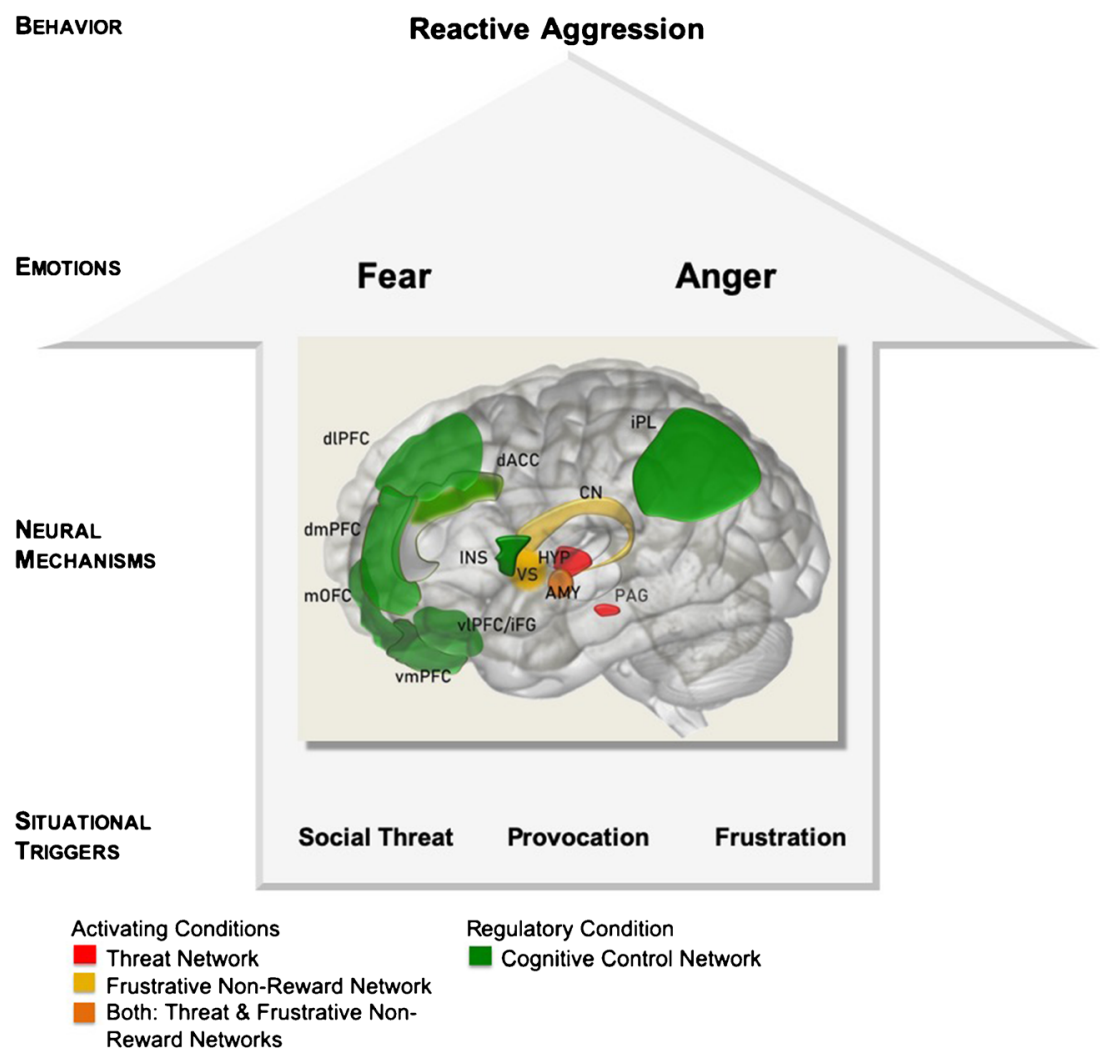


provoke anger and retaliatory aggression were found to activate the threat circuit, provocation might be regarded as a specific form of social threat. In individuals with a predisposed sensitivity to provocation and poor (lateral) prefrontal emotion regulation capacities, as often found in clinical groups, provocation elicits strong feelings of anger, which in the case of deficient inferior fronto-parietal inhibitory control escalates into aggressive reactions, even in response to minor provocations [89]. Furthermore, social threat processing can interact with the evaluation of value and contingency (vmPFC), i.e., aggressive responses are less likely if the situational context, the aggressive action itself, and its consequences are not expected to provide reward. Furthermore, abnormal threat processing can interact with frustrative non-reward, as has been shown in irritable youth [90]. More knowledge on the rewarding nature of aggressive outbursts is needed. This is of particular relevance with regard to frustration, another prominent trigger for aggression. Although it remains difficult to distinguish its psychological and neurobiological effects from those of provocation, e.g., because both provoke a tit-for-tat-like retaliatory aggression. Other than povocation, frustration is closely associated with prediction errors and the brain's reward system, with higher VS activity correlating with or predicting the degree of realiatory behavior particularly in individuals with low frustration tolerance [35, 41]. Retaliatory aggression following frustration may involve inadequate processing of response costs and abnormalities in reward and threat processing associated with dysfunctions in the VS [41] and the threat system [47], and reduced vmPFC-amygdala connectivity [34-] as seen when highly reactively aggressive clinical groups punish unfair opponents. Furthermore, reactive aggression following frustrative non-reward is modulated by regions associated with evaluation, and particularly theory-of-mind processes and thus the awareness of one's own and other people's intentions. This is in line with the social information processing theory of aggression [91], according to which frustration only results in aggressive retaliation when the frustrated individual attributes hostile intent to the opponent. Furthermore, decreased empathy, i.e., reduced sharing of other people's negative emotions, and reduced compassion were reported in aggressive offenders [92]. Overlap also exists between frustrative non-reward and acute threat which, in the Research Domain Criteria (RDoC) matrix [93] both belong to the negative valence system and have been related to the same paradigms with blocking of an expected access to resources (e.g., competitive games, ostracism, or unfair treatment) as these may induce both frustration and threat. Evidence for a difference between these systems comes from studies that found competitive processes between regions responding to threat of shock and those engaged in decisionmaking about a monetary reward [94]. In irritable youths, threat hypersensitivity and frustrative non-reward are thought to interact [90].

As noted above, the current literature leaves open several important research questions that should be addressed in future studies. First, most of the currently used paradigms are not designed in a way to disentangle distinct motivations for aggressive behavior as proposed by the traditional differentiation between threat, provocation, and frustration [3]. We do not know whether hyperactivation of the basic threat circuit indeed reflects a specific threat response or rather a socially challenging and emotionally salient event in general [95] that is processed in the amygdala and affects physiology and behavior via downstream activations of hypothalamus and PAG [96]. This challenges the question whether provocation which has been shown to activate the threat circuit actually reflects a complex form of social threat to humans or just like social threat acts as a salient event. Thus, provocation, e.g., in the shape of unfair offers, may be perceived as harmful or as a salient event pointing to expectancy violation which results in frustration. To answer this question, more complex paradigms have to be developed in which the adaptiveness of social decision strategies can be quantified and used for computational modeling. Thereby, paradigms could be developed that allow a differentiation between threat and provocation, e.g., by varying the intentions of a co-player [97]. Second, it is also almost impossible to disentangle brain circuits related to frustrative nonreward from those related to provocation in commonly used economic game paradigms, since they measure retaliation in response to unfair and thus provocative offers that frustrate the participants' reward expectancies. Economic game paradigms are the most frequently selected tasks to investigate frustration. While in these tasks, frustration is usually provided by human opponents, we do not know how aggression is related to non-social frustration (e.g., in case the PC does not work appropriately). Furthermore, both provocation and frustration elicit anger, which, however, may either result in explosive outbursts or in retaliatory behavior. Notably, the latter can be of impulsive or premediated, instrumental nature: While impulsive retaliation is likely associated with inadequate processing of response costs, premediated retaliation is rather an instrumental, intentionally selected response, congruent with reinforcement expectancies [3]. Consequently, future paradigms need to challenge impulsive as opposed to premediated retaliatory aggression within one study design. Third, we do not know yet whether threat-related aggression is directly provoked by the emotion of fear as suggested by the animal model of defensive aggression or implicates a move from avoiding toward approaching threat that is inseparably linked to a switch from fear to anger. fMRI tasks of social threat that combine the measurement of aggression level with queries on accompanying emotions of anger and fear may contribute to answer this issue. Forth, based on animal results, it might be interesting to study whether reactive aggression is associated 
with aberrant perceptions of the proximity of a threat in future studies. To our knowledge, this has not been addressed in humans, yet, despite the availability of MRI paradigms which allow the manipulation of threat proximity [98]. Fifth, it remains difficult to disentangle the involvement of specific cognitive control functions in the regulation of reactive aggression based on the current literature. Tight interactions between behavior and emotion control have been described and first results in healthy participants suggest that problems in emotion regulation might be particularly problematic when individuals with low inhibitory control are facing threats, provocations, or frustrations [73]. Finally and more generally, further effort is needed regarding the measurement of reactive aggression. Aside from valid reports about real-life aggression, it is necessary to develop new, ecologically more valid experimental paradigms that systematically manipulate social context information and include behavioral options other than aggression. Moreover, technological advances in high-resolution fMRI and machine learning should be utilized in order to better tackle the complex neural mechanisms of reactive aggression and to further disentangle specific psychological and neural mechanisms of reactive aggression. This would provide the chance to develop mechanism-based treatments that specifically address the individual patient's needs, given that so far, treatment approaches have only shown small effect sizes $[99,100]$. A detailed diagnostic assessment including experimental measures of threat sensitivity, frustrative non-reward, and cognitive control could be helpful for implementing modularized psychotherapy and targeted pharmacotherapy.

\section{Conclusions}

For clinical samples with high aggression and prominent threat sensitivity, improving prefrontal-amygdala inhibition in response to alleged threats, provocations, and frustrations might be a promising target for interventions. Correspondingly, activations in social threat and emotion control circuits have already been shown to be susceptible to psychotherapy [101], brain stimulation [42,61], and neurofeedback [102]. However, more specific interventions are needed to target the specific mechanisms underlying reactive aggression in this population (Herpertz et al., under review).

Supplementary Information The online version contains supplementary material available at https://doi.org/10.1007/s11920-020-01208-6.

Acknowledgments The editors would like to thank Dr. Christan Schmahl for taking the time to review this manuscript.

Funding Open Access funding enabled and organized by Projekt DEAL. Katja Bertsch and Sabine C. Herpertz received funding from the German
Research Foundation (Clinical Research Group 256 (KFO-256, BE5292/ 2-1, BE5292/3-2, HE 2660/12-1, HE 2660/12-2). Sabine C. Herpertz's research work was supported by the European Union FP7-Health Program Grant (FemNAT-CD 602407).

\section{Compliance with Ethical Standards}

Conflict of Interest Katja Bertsch, Julian Florange, and Sabine C. Herpertz declare that they have no conflict of interest.

Human and Animal Rights and Informed Consent This article does not contain any studies with human or animal subjects performed by any of the authors.

Open Access This article is licensed under a Creative Commons Attribution 4.0 International License, which permits use, sharing, adaptation, distribution and reproduction in any medium or format, as long as you give appropriate credit to the original author(s) and the source, provide a link to the Creative Commons licence, and indicate if changes were made. The images or other third party material in this article are included in the article's Creative Commons licence, unless indicated otherwise in a credit line to the material. If material is not included in the article's Creative Commons licence and your intended use is not permitted by statutory regulation or exceeds the permitted use, you will need to obtain permission directly from the copyright holder. To view a copy of this licence, visit http://creativecommons.org/licenses/by/4.0/.

\section{References}

Papers of particular interest, published recently, have been highlighted as:

- Of importance

1. Baron RA, Richardson DR. Human Aggression. New York: Plenum; 1994.

2. Berkowitz L. Pain and aggression: some findings and implications. Motiv Emot. 1993;17(3):277-93.

3. Blair RJR. Traits of empathy and anger: implications for psychopathy and other disorders associated with aggression. Philos Trans R Soc Lond B Biol Sci. 2018 Apr 19;373(1744):20170155. https://doi.org/10.1098/rstb.2017.0155

4. Verona E, Bresin K. Aggression proneness: transdiagnostic processes involving negative valence and cognitive systems. Int $\mathrm{J}$ Psychophysiol. 2015;98(2 Pt 2):321-9.

5. Amsel A. Précis offrustration theory: an analysis of dispositional learning and memory. Psychon Bull Rev. 1994;1(3):280-96.

6. Gatzke-Kopp LM, Willner CJ, Jetha MK, Abenavoli RM, DuPuis D, Segalowitz SJ. How does reactivity to frustrative non-reward increase risk for externalizing symptoms? Int J Psychophysiol. 2015;98(2 Pt 2):300-9.

7. Fernandez KC, Jazaieri H, Gross JJ. Emotion regulation: a transdiagnostic perspective on a new RDoC domain. Cogn Ther Res. 2016;40(3):426-40.

8. Carver CS, Harmon-Jones E. Anger is an approach-related affect: evidence and implications. Psychol Bull. 2009;135(2):183-204.

9. Blanchard DC, Hynd AL, Minke KA, Minemoto T, Blanchard RJ. Human defensive behaviors to threat scenarios show parallels to fear- and anxiety-related defense patterns of non-human mammals. Neurosci Biobehav Rev. 2001;25(7-8):761-70. 
10. Blanchard DC. Translating dynamic defense patterns from rodents to people. Neurosci Biobehav Rev. 2017;76:22-8.

11. Blanchard DC, Blanchard RJ. What can animal aggression research tell us about human aggression? Horm Behav. 2003;44(3):171-7.

12. Haller J. The role of central and medial amygdala in normal and abnormal aggression: a review of classical approaches. Neurosci Biobehav Rev. 2018;85:34-43.

13. Toth M, Tulogdi A, Biro L, Soros P, Mikics E, Haller J. The neural background of hyper-emotional aggression induced by post-weaning social isolation. Behav Brain Res. 2012;233(1): 120-9.

14. Beyer F, Buades-Rotger M, Claes M, Kramer UM. Hit or run: exploring aggressive and avoidant reactions to interpersonal provocation using a novel fight-or-escape paradigm (FOE). Front Behav Neurosci. 2017;11:190.

15. Gunderson JG, Herpertz SC, Skodol AE, Torgersen S, Zanarini MC. Borderline personality disorder. Nat Rev Dis Primers. 2018;4:18029.

16. Bertsch K, Gamer M, Schmidt B, Schmidinger I, Walther S, Kastel T, et al. Oxytocin and reduction of social threat hypersensitivity in women with borderline personality disorder. Am J Psychiatry. 2013;170(10):1169-77.

17. Smeijers D, Rinck M, Bulten E, van den Heuvel T, Verkes RJ. Generalized hostile interpretation bias regarding facial expressions: characteristic of pathological aggressive behavior. Aggress Behav. 2017;43(4):386-97.

18. Teicher MH, Samson JA. Childhood maltreatment and psychopathology: a case for ecophenotypic variants as clinically and neurobiologically distinct subtypes. Am J Psychiatry. 2013;170(10):1114-33.

19. Blair JR. The neurobiology of disruptive behavior disorder. Am J Psychiatry. 2016;173(11):1073-4.

20. Teicher MH, Samson JA, Anderson CM, Ohashi K. The effects of childhood maltreatment on brain structure, function and connectivity. Nat Rev Neurosci. 2016;17(10):652-66.

21. Farah T, Ling S, Raine A, Yang Y, Schug R. Alexithymia and reactive aggression: the role of the amygdala. Psychiatry Res Neuroimaging. 2018;281:85-91.

22. Coccaro EF, Fitzgerald DA, Lee R, McCloskey M, Phan KL. Frontolimbic morphometric abnormalities in intermittent explosive disorder and aggression. Biol Psychiatry Cogn Neurosci Neuroimaging. 2016;1(1):32-8.

23. Coccaro EF, Lee R, McCloskey M, Csernansky JG, Wang L. Morphometric analysis of amygdala and hippocampus shape in impulsively aggressive and healthy control subjects. J Psychiatr Res. 2015;69:80-6.

24. Mancke F, Herpertz SC, Hirjak D, Knies R, Bertsch K. Amygdala structure and aggressiveness in borderline personality disorder. Eur Arch Psychiatry Clin Neurosci. 2018;268(4):417-27.

25. Reed LI, DeScioli P, Pinker SA. The commitment function of angry facial expressions. Psychol Sci. 2014;25(8):1511-7.

26. Ashley V, Swick D. Angry and fearful face conflict effects in posttraumatic stress disorder. Front Psychol. 2019;10:136.

27. Choe DE, Shaw DS, Forbes EE. Maladaptive social information processing in childhood predicts young men's atypical amygdala reactivity to threat. J Child Psychol Psychiatry. 2015;56(5):549 57 This longditudinal study revealed that impaired social information processing at ages 10-11 years predicts increased amygdala reactivity to fearful faces at age 20 years in $\mathbf{3 1 0}$ individuals.

28. Buades-Rotger M, Engelke C, Beyer F, Keevil BG, Brabant G, Kramer UM. Endogenous testosterone is associated with lower amygdala reactivity to angry faces and reduced aggressive behavior in healthy young women. Sci Rep. 2016;6:38538.
29. Klimecki OM, Sander D, Vuilleumier P. Distinct brain areas involved in anger versus punishment during social interactions. Sci Rep. 2018;8(1):10556.

30. McCloskey MS, Phan KL, Angstadt M, Fettich KC, Keedy S, Coccaro EF. Amygdala hyperactivation to angry faces in intermittent explosive disorder. J Psychiatr Res. 2016;79:34-41.

31. Bertsch K, Krauch M, Stopfer K, Haeussler K, Herpertz SC, Gamer M. Interpersonal threat sensitivity in borderline personality disorder: an eye-tracking study. J Personal Disord. 2017;31(5): 647-70.

32. da Cunha-Bang S, Fisher PM, Hjordt LV, Holst K, Knudsen GM Amygdala reactivity to fearful faces correlates positively with impulsive aggression. Soc Neurosci. 2019;14(2):162-72.

33. White SF, Brislin SJ, Sinclair S, Blair JR. Punishing unfairness: rewarding or the organization of a reactively aggressive response? Hum Brain Mapp. 2014;35(5):2137-47.

34. White SF, VanTieghem M, Brislin SJ, Sypher I, Sinclair S, Pine DS, et al. Neural correlates of the propensity for retaliatory behavior in youths with disruptive behavior disorders. Am J Psychiatry. 2016;173(3):282-90 This is one of the few published functional MRI studies in a large group of pathologically aggressive adolescents.

35. da Cunha-Bang S, Fisher PM, Hjordt LV, Perfalk E, Persson Skibsted A, Bock C, et al. Violent offenders respond to provocations with high amygdala and striatal reactivity. Soc Cogn Affect Neurosci. 2017;12(5):802-10.

36. Fanning JR, Keedy S, Berman ME, Lee R, Coccaro EF. Neural correlates of aggressive behavior in real time: a review of fMRI studies of laboratory reactive aggression. Curr Behav Neurosci Rep. 2017;4(2):138-50.

37. Siegrist J, Menrath I, Stocker T, Klein M, Kellermann T, Shah NJ, et al. Differential brain activation according to chronic social reward frustration. Neuroreport. 2005;16(17):1899-903.

38. Leibenluft E. Pediatric irritability: a systems neuroscience approach. Trends Cogn Sci. 2017;21(4):277-89.

39. FitzGerald TH, Schwartenbeck P, Dolan RJ. Reward-related activity in ventral striatum is action contingent and modulated by behavioral relevance. J Neurosci. 2014;34(4):1271-9.

40. Berkowitz L. Frustration-aggression hypothesis: examination and reformulation. Psychol Bull. 1989;106(1):59-73.

41. Chester DS, DeWall CN. The pleasure of revenge: retaliatory aggression arises from a neural imbalance toward reward. Soc Cogn Affect Neurosci. 2016;11(7):1173-82.

42. Gilam G, Abend R, Gurevitch G, Erdman A, Baker H, Ben-Zion $\mathrm{Z}$, et al. Attenuating anger and aggression with neuromodulation of the vmPFC: a simultaneous tDCS-fMRI study. Cortex. 2018;109:156-70.

43. Chester DS, Lynam DR, Milich R, DeWall CN. Physical aggressiveness and gray matter deficits in ventromedial prefrontal cortex. Cortex. 2017;97:17-22.

44. Yang Y, Joshi SH, Jahanshad N, Thompson PM, Baker LA. Neural correlates of proactive and reactive aggression in adolescent twins. Aggress Behav. 2017;43(3):230-40.

45. Chester DS, Lynam DR, Milich R, DeWall CN. Neural mechanisms of the rejection-aggression link. Soc Cogn Affect Neurosci. 2018;13(5):501-12.

46. White SF, Pope K, Sinclair S, Fowler KA, Brislin SJ, Williams WC, et al. Disrupted expected value and prediction error signaling in youths with disruptive behavior disorders during a passive avoidance task. Am J Psychiatry. 2013;170(3):315-23 This study reports important results with regard to sensitivity of the ventromedial prefontal cortex to expected value processing in pathologically aggressive adolescents.

47. Deveney CM, Connolly ME, Haring CT, Bones BL, Reynolds RC, Kim P, et al. Neural mechanisms of frustration in chronically irritable children. Am J Psychiatry. 2013;170(10):1186-94. 
48. Miller EK, Cohen JD. An integrative theory of prefrontal cortex function. Annu Rev Neurosci. 2001;24:167-202.

49. Framework DotR. National institute of mental health. 2016.

50. Puiu AA, Wudarczyk O, Goerlich KS, Votinov M, HerpertzDahlmann B, Turetsky B, et al. Impulsive aggression and response inhibition in attention-deficit/hyperactivity disorder and disruptive behavioral disorders: findings from a systematic review. Neurosci Biobehav Rev. 2018;90:231-46.

51. Sebastian A, Retz W, Tuscher O, Turner D. Violent offending in borderline personality disorder and attention deficit/hyperactivity disorder. Neuropharmacology. 2019;156:107565.

52. McRae K, Gross JJ. Emotion regulation. Emotion. 2020;20(1):1-9.

53. Hofhansel L, Weidler C, Votinov M, Clemens B, Raine A, Habel U. Morphology of the criminal brain: gray matter reductions are linked to antisocial behavior in offenders. Brain Struct Funct. 2020;225:2017-28.

54. Beyer F, Munte TF, Gottlich M, Kramer UM. Orbitofrontal cortex reactivity to angry facial expression in a social interaction correlates with aggressive behavior. Cereb Cortex. 2015;25(9):305763.

55. Ochsner, K. N., \& Gross, J. J. (2014). The neural bases of emotion and emotion regulation: A valuation perspective. In J. J. Gross (Ed.), Handbook of emotion regulation (p. 23-42). The Guilford Press.

56. Dalley JW, Robbins TW. Fractionating impulsivity: neuropsychiatric implications. Nat Rev Neurosci. 2017;18(3):158-71.

57. Bertsch K, Roelofs K, Roch PJ, Ma B, Hensel S, Herpertz SC, et al. Neural correlates of emotional action control in anger-prone women with borderline personality disorder. J Psychiatry Neurosci. 2018;43(3):161-70 This study revealed reduced prefrontal-amygdala connectivity in anger-prone women with borderline personality disorder while approaching angry faces - a result which is similar to previous findings in highly aggressive male offenders.

58. Bertsch K, Krauch M, Roelofs K, Cackowski S, Herpertz SC, Volman I. Out of control? Acting out anger is associated with deficient prefrontal emotional action control in male patients with borderline personality disorder. Neuropharmacology. 2019;156: 107463 This study replicates and extends previous report of reduced prefrontal and elevated amygdala activations during the approach of angry faces in male patients with borderline personality disorder. It is one of the few studies focussing on male patients with borderline personality disroder.

59. Volman I, von Borries AK, Bulten BH, Verkes RJ, Toni I, Roelofs K. Testosterone Modulates Altered Prefrontal Control of Emotional Actions in Psychopathic Offenders $(1,2,3)$. eNeuro. 2016;8;3(1). https://doi.org/10.1523/ENEURO.0107-15.2016

60. Kaldewaij R, Koch SB, Volman I, Toni I, Roelofs K. On the control of social approach-avoidance behavior: neural and endocrine mechanisms. Curr Top Behav Neurosci. 2017;30:275-93.

61. Volman I, Roelofs K, Koch S, Verhagen L, Toni I. Anterior prefrontal cortex inhibition impairs control over social emotional actions. Curr Biol. 2011;21(20):1766-70.

62. Dorfel D, Lamke JP, Hummel F, Wagner U, Erk S, Walter H. Common and differential neural networks of emotion regulation by Detachment, Reinterpretation, Distraction, and Expressive Suppression: a comparative fMRI investigation. Neuroimage. 2014;101:298-309. https://doi.org/10.1016/j.neuroimage.2014. 06.051

63. Wager TD, Smith EE. Neuroimaging studies of working memory: a meta-analysis. Cogn Affect Behav Neurosci. 2003;3(4):255-74.

64. Achterberg M, van Duijvenvoorde ACK, Bakermans-Kranenburg MJ, Crone EA. Control your anger! The neural basis of aggression regulation in response to negative social feedback. Soc Cogn Affect Neurosci. 2016;11(5):712-20.
65. Herpertz SC, Nagy K, Ueltzhoffer K, Schmitt R, Mancke F, Schmahl $\mathrm{C}$, et al. Brain mechanisms underlying reactive aggression in borderline personality disorder-sex matters. Biol Psychiatry. 2017;82(4):257-66 This study investigated neural correlates of anger and aggression in a relatively large sample of female and male patients with borderline personality disorder. It is one of the few studies addressing sex-dependent neural alterations in borderline personality disorder associated with aggression.

66. Ueltzhöffer K, Herpertz SC, Krauch M, Schmahl C, Bertsch K. Whole-brain functional connectivity during script-driven aggression in borderline personality disorder. Prog NeuroPsychopharmacol Biol Psychiatry. 2019;93:46-54.

67. Buades-Rotger M, Engelke C, Kramer UM. Trait and state patterns of basolateral amygdala connectivity at rest are related to endogenous testosterone and aggression in healthy young women. Brain Imaging Behav. 2019;13(2):564-76.

68. Chester DS, Bell SB, DeWall CN, West SJ, Romero-Lopez M, Craig AW. Neural correlates of intertemporal choice in aggressive behavior. Aggress Behav. 2019;45(5):507-16.

69. Tateno A, Jorge RE, Robinson RG. Clinical correlates of aggressive behavior after traumatic brain injury. J Neuropsychiatry Clin Neurosci. 2003;15(2):155-60.

70. Reddy KJ, Menon KR, Hunjan UG. Neurobioloigical aspects of violent and criminal behaviour: deficits in frontal lobe function and neurotransmitters. Int J Crim Justice Sci. 2018;13(1):44-54.

71. Xue J-M, Lin P-Z, Sun J-W, Cao F-L. Disrupted executive function and aggression in individuals with a history of adverse childhood experiences: an event-related potential study. J Nerv Ment Dis. 2017;205:942-51.

72. Lievaart M, van der Veen FM, Huijding J, Hovens JE, Franken IHA. The relation between trait anger and impulse control in forensic psychiatric patients: an EEG study. Appl Psychophysiol Biofeedback. 2018:43:131-42.

73. Chester DS, Eisenberger NI, Pond RS, Richman SB, Bushman BJ, DeWall CN. The interactive effect of social pain and executive functioning on aggression: an fMRI experiment. Soc Cogn Affect Neurosci. 2014;9:699-704.

74. Deng W, Rolls ET, Ji X, Robbins TW, Banaschewski T, Bokde ALW, et al. Separate neural systems for behavioral change and for emotional responses to failure during behavioral inhibition. Hum Brain Mapp. 2017;38(7):3527-37.

75. Gore WL, Widiger TA. The DSM-5 dimensional trait model and five-factor models of general personality. J Abnorm Psychol. 2013;122(3):816-21.

76. McClure G, Hawes DJ, Dadds MR. Borderline personality disorder and neuropsychological measures of executive function: a systematic review. Personal Ment Health. 2016;10(1):43-57.

77. McHugh C, Balaratnasingam S. Impulsivity in personality disorders: current views and future directions. Curr Opin Psychiatry. 2018;31(1):63-8.

78. Scott LN, Stepp SD, Pilkonis PA. Prospective associations between features of borderline personality disorder, emotion dysregulation, and aggression. Personal Disord. 2014;5(3):278-88.

79. Garofalo C, Neumann CS, Velotti P. Difficulties in emotion regulation and psychopathic traits in violent offenders. J Crim Just. 2018:57:116-25.

80. Mancke F, Herpertz SC, Kleindienst N, Bertsch K. Emotion dysregulation and trait anger sequentially mediate the association between borderline personality disorder and aggression. J Personal Disord. 2017;31(2):256-72.

81. Ahmed AO, Richardson J, Buckner A, Romanoff S, Feder M, Oragunye N, et al. Do cognitive deficits predict negative emotionality and aggression in schizophrenia? Psychiatry Res. 2018;259:350-7. 
82. Lickley RA, Sebastian CL. The neural basis of reactive aggression and its development in adolescence. Psychol Crime Law. 2018;24(3):313-33.

83. Waltes R, Chiocchetti AG, Freitag CM. The neurobiological basis of human aggression: a review on genetic and epigenetic mechanisms. Am J Med Genet B Neuropsychiatr Genet. 2016;171(5): 650-75.

84. Denson TF, O'Dean SM, Blake KR, Beames JR. Aggression in women: behavior, brain and hormones. Front Behav Neurosci. 2018;12:81.

85. Heany SJ, Bethlehem RAI, van Honk J, Bos PA, Stein DJ, Terburg D. Effects of testosterone administration on threat and escape anticipation in the orbitofrontal cortex. Psychoneuroendocrinology. 2018;96:42-51.

86. Geniole SN, Procyshyn TL, Marley N, Ortiz TL, Bird BM, Marcellus AL, et al. Using a psychopharmacogenetic approach to identify the pathways through which - and the people for whom - testosterone promotes aggression. Psychol Sci. 2019;30(4):48194.

87. Tackett JL, Reardon KW, Herzhoff K, Page-Gould E, Harden KP, Josephs RA. Estradiol and cortisol interaction in youth externalizing psychopathology. Psychoneuroendocrinology. 2015;55:14653.

88. Timmermann M, Jeung H, Schmitt R, Boll S, Freitag CM, Bertsch K, et al. Oxytocin improves facial emotion recognition in young adults with antisocial personality disorder. Psychoneuroendocrinology. 2017;85:158-64.

89. Fettich KC, McCloskey MS, Look AE, Coccaro EF. Social cognition in intermittent explosive disorder and aggression. J Psychiatr Res. 2016;83:140-50.

90. Brotman MA, Kircanski K, Stringaris A, Pine DS, Leibenluft E. Irritability in youths: a translational model. Am J Psychiatry. 2017;174(6):520-32.

91. Crick NR, Dodge KA. Social information-processing mechanisms in reactive and proactive aggression. Child Dev. 1996;67(3):9931002.

92. Winter K, Spengler S, Bermpohl F, Singer T, Kanske P. Social cognition in aggressive offenders: impaired empathy, but intact theory of mind. Sci Rep. 2017;7(1):670.
93. Insel T, Cuthbert B, Garvey M, Heinssen R, Pine DS, Quinn K, et al. Research domain criteria (RDoC): toward a new classification framework for research on mental disorders. Am J Psychiatry. 2010;167(7):748-51.

94. Choi JM, Padmala S, Spechler P, Pessoa L. Pervasive competition between threat and reward in the brain. Soc Cogn Affect Neurosci. 2014;9(6):737-50.

95. Jonas E, Mcgregor JE, Klack1 J, Agroskin D, Fritsche I, Holbrook C, Nash K, Proulx T, Quirin M (2014). Threat and defense: From anxiety to approach. In J. M. Olson, \& M. P. Zanna (Eds.), Advances in experimental social psychology (Vol. 49, pp. 219 286). Academic Press. https://doi.org/10.1016/B978-0-12800052-6.00004-4

96. O'Neill PK, Gore F, Salzman CD. Basolateral amygdala circuitry in positive and negative valence. Curr Opin Neurobiol. 2018;49: 175-83.

97. Korn CW, Bach DR. Minimizing threat via heuristic and optimal policies recruits hippocampus and medial prefrontal cortex. Nat Hum Behav. 2019;3(7):733-45.

98. Montoya ER, van Honk J, Bos PA, Terburg D. Dissociated neural effects of cortisol depending on threat escapability. Hum Brain Mapp. 2015;36(11):4304-16.

99. Lee AH, DiGiuseppe R. Anger and aggression treatments: a review of meta-analyses. Curr Opin Psychol. 2018;19:65-74.

100. Hendriks AM, Bartels M, Colins OF, Finkenauer C. Childhood aggression: a synthesis of reviews and meta-analyses to reveal patterns and opportunities for prevention and intervention strategies. Neurosci Biobehav Rev. 2018;91:278-91.

101. Schmitt F, Stieb SM, Wehner R, Rossler W. Experience-related reorganization of giant synapses in the lateral complex: potential role in plasticity of the sky-compass pathway in the desert ant Cataglyphis fortis. Dev Neurobiol. 2016;76(4):390-404.

102. Paret C, Kluetsch R, Zaehringer J, Ruf M, Demirakca T, Bohus M, et al. Alterations of amygdala-prefrontal connectivity with realtime fMRI neurofeedback in BPD patients. Soc Cogn Affect Neurosci. 2016;11:952-60.

Publisher's Note Springer Nature remains neutral with regard to jurisdictional claims in published maps and institutional affiliations. 